\title{
Planetary waves and vortex structures in the ionosphere
}

\author{
A. G. Khantadze ${ }^{1}$, G. V. Jandieri ${ }^{2}$, A. Ishimaru ${ }^{3}$, and V. G. Jandieri ${ }^{4}$ \\ ${ }^{1}$ Tbilisi State University, 1 Chavchavadze ave., 0128, Tbilisi, Georgia \\ ${ }^{2}$ Physics Department, Georgian Technical University, 77 Kostava str, 0179, Tbilisi, Georgia \\ ${ }^{3}$ Department of Electrical Engineering, University of Washington, FT-10 Seattle, WA 98195, USA \\ ${ }^{4}$ Department of Electrical and Computer Engineering, Kumamoto University, Japan
}

Received: 16 May 2009 - Revised: 7 August 2009 - Accepted: 26 August 2009 - Published: 22 September 2009

\begin{abstract}
The problem of propagation of large-scale vortices in the ionosphere is investigated. Permanently acting fundamental factors have been discovered making it possible to reveal the mechanisms of generation of large-scale vortices and planetary waves in conductive atmosphere.
\end{abstract}

Keywords. Radio science (Ionospheric physics; Ionospheric propagation; Waves in plasma)

\section{Introduction}

Recently a problem in the study of dynamics of large-scale $\left(10^{3}-10^{4} \mathrm{~km}\right)$ motions in the ionosphere, against the background of which almost all physicochemical processes occur, is the focus of the research investigating the upper atmosphere. This is caused by the fact that at the considered altitudes $(80 \div 600 \mathrm{~km})$, atmosphere is weakly ionized ionospheric plasma - the charged component of which instantly reacts to any changes of the dynamic mode of the neutral component of ionosphere. The response to the dynamic impact has electromagnetic character. It propagates in the medium with a velocity over $1 \mathrm{~km} / \mathrm{s}$ in the form of natural (short-period) oscillations, from several minutes to several hours and with a wavelength from several meters up to $10^{3} \mathrm{~km}$. Ionospheric parameters in the E- and F-regions have also long-period oscillations with characteristic periods from two days to two weeks and longer. Also, if we take into account that the horizontal spatial scale (of wavelength) of these oscillations is $10^{3}-10^{4} \mathrm{~km}$ it can be assumed that such variations of ionospheric parameters reflect the "response" of plasma when the planetary waves are passing in the upper atmosphere. According to the theory (Charney and Drazin, 1961; Dickinson, 1968), propagation of planetary waves from lower atmosphere into the upper at-

\section{Correspondence to: G. V. Jandieri} (jandieri@access.sanet.ge) mosphere depends on the magnitudes of the waves themselves as well as on parameters of general circulation at this level of atmosphere. In particular, for the condition of propagation of planetary waves up simple inequality is obtained $k(k+1)<2 \omega_{0} / \alpha$, where $k$ - wave number, $\alpha$ - circulation index, $\omega_{0}$ - angular velocity of the Earth's rotation. In the troposphere, zonal winds are directed to the east in summer $(\alpha<0)$, and to the west in winter $(\alpha>0)$. Consequently, in summer there are no conditions for their propagation as in such case inequality is violated. In winter only the largest-scale perturbations can propagate up with wave numbers $k=1$ and 2 (usually $\left(\omega_{0} / \alpha\right) \approx 4$ ). Thus, penetration of planetary waves with wave numbers $k>2$ is theoretically impossible. However, experimental results of an investigation of long-period variations of ionospheric parameters show that "response" to the passage of planetary waves in the ionosphere are observed not only in winter but also in summer, besides variations with wave numbers $k>2$ are observed. All the above shows that the sources of planetary waves and large-scale vortices can exist, not only in the lower atmosphere but also in the ionosphere itself (Sharadze et al., 1989). The response of ionospheric plasma to the passage of waves in the upper atmosphere is usually registered by the ionospheric and magnetic observatories during magnetic storms, substorms, earthquakes, launch of spacecrafts (Burmaka et al., 2003), etc. The interpretation of the response of ionospheric plasma is a vital task of researchers of the upper atmosphere and the near-earth space. Dynamics of the ionospheric cyclones and anticyclones corresponding to maxima and minima of the planetary waves analytically is investigated in Petviashvili et al. (1993). The acoustic gravity waves in the ionosphere generating meteorological noise for the planetary waves are considered in Nekrasov et al. (1995) and Kaladze et al. (2008).

The dynamics of planetary waves and large-scale vortex structures in the ionosphere is analytically investigated in this paper. 


\section{Model of medium and basic equations}

Characteristics of the dynamic processes in the upper atmosphere are determined by the presence of an electroconductive component and by the effect of the geomagnetic field on this component. The presence of anisotropic conductivity and an inhomogeneous geomagnetic field gives additional electromagnetic elasticity to the upper atmosphere of the earth. The closed system of equations of the magnetic hydrodynamics of ionosphere with a given heat flow $\varepsilon$, can be presented in the following form (Khantadze, 1973):

$$
\rho \frac{d \boldsymbol{V}}{d t}=-\nabla P+\rho \boldsymbol{g}+\rho\left[\boldsymbol{V} \times 2 \boldsymbol{\omega}_{\mathbf{0}}\right]+\frac{1}{4 \pi}\left[\nabla \times \boldsymbol{h} \times \boldsymbol{H}_{\mathbf{0}}\right]
$$

$$
\begin{aligned}
\frac{\partial \boldsymbol{h}}{\partial t}= & \nabla \times\left[\boldsymbol{V} \times \boldsymbol{H}_{\mathbf{0}}\right]-\delta \nabla \times \frac{\alpha}{4 \pi}\left[\nabla \times \boldsymbol{h} \times \boldsymbol{H}_{\mathbf{0}}\right]+\nabla \\
& \times\left[\boldsymbol{H}_{\mathbf{0}} \frac{1}{\rho_{i} v_{i m}} \frac{1}{4 \pi}\left[\nabla \times \boldsymbol{h} \times \boldsymbol{H}_{\mathbf{0}}\right]\right] .
\end{aligned}
$$

$\frac{\partial \rho}{\partial t}+\nabla \cdot(\rho \boldsymbol{V})=0$

$$
\frac{d P}{d t}+\gamma P \nabla \cdot V=\varepsilon
$$

Here $\boldsymbol{V}$ and $\boldsymbol{H}=\boldsymbol{H}_{\mathbf{0}}+\boldsymbol{h}$ - velocity and magnetic field, $\boldsymbol{H}_{\mathbf{0}}-$ geomagnetic field, $h$-deviation from $\boldsymbol{H}_{\mathbf{0}}, P$ and $\rho=M N_{m}$, $P$ and $\rho=M N_{m}$ - pressure and density of neutrals, $\rho_{i}=M_{i} N_{i}$ - density of ions, $N$ and $N_{n}$ - plasma and neutrals densities, $v_{i m}$ - collision frequency of ions with neutrals, $\boldsymbol{g}$ - acceleration of gravity, $\omega_{0}$ - angular velocity of the Earth's rotation, $\alpha=c / e N-$ Hall parameter, $\gamma$ - polytropic index. The dimensionless parameter $\delta$ is introduced for convenience: in the Eregion $(80-150 \mathrm{~km}) \delta=1$, and in the F-region $(150-600 \mathrm{~km})$ $\delta=0$.

For the E-region, taking "rot" from both parts of Eq. (1) and adding it to Eq. (2) - on condition of incompressibility and absence of dissipative forces - we will find the condition of conservation of the new invariant (Khantadze, 1972):

$\operatorname{helm}\left(\nabla \times \boldsymbol{V}+2 \omega_{\mathbf{0}}+\frac{N}{N_{m}} \frac{e}{M c} \boldsymbol{H}_{\mathbf{0}}\right)=0$.

Here, the operator "helm" for any vector field $\boldsymbol{a}$ has the following form:

helm $\boldsymbol{a}=\frac{\partial \boldsymbol{a}}{\partial t}-\nabla \times[\boldsymbol{V} \times \boldsymbol{a}]+\boldsymbol{V} \nabla \cdot \boldsymbol{a}$.

Equality helm $\boldsymbol{a}=0$ means conservation ("freezing-in") of both, force lines of vector $\boldsymbol{a}$ and intensity of vector tubes. In the absence of the magnetic field $\left(\boldsymbol{H}_{\mathbf{0}}=0\right)$ from Eq. (5), we obtain the well-known conservation condition ("freezingin") of the absolute vortex $\nabla \times \boldsymbol{V}+2 \omega_{0}$ (Pedloskii, 1984) which, as a particular case, contains slow weather-forming planetary Rossby waves caused by latitudinal inhomogeneity of angular velocity of the earth rotation $\nabla \omega_{0} \neq 0$. In the minimums and maximums of planetary waves, there are always tropospheric cyclones and anticyclones located, together with the wave propagate with the velocity of a medium zonal wind $\left(\sim 10 \mathrm{~m} \mathrm{~s}^{-1}\right)$ and actually determine the regional weather in the atmosphere of the Earth. From Eq. (5), it follows that slow planetary waves must exist in the lower ionospheric E-region, caused by latitudinal inhomogeneities of $\nabla \omega_{0}$ and $\nabla H_{0}$. Equation (5) shows partial freezing-in of the absolute vortex. When $H_{0} \rightarrow 0$ (Eq. 5) they change to Fridman's equation for the absolute vortex $\nabla \times \boldsymbol{V}+2 \boldsymbol{\omega}_{\mathbf{0}}$, and when $H_{0} \rightarrow 0$ and $\nabla \omega_{0} \rightarrow 0$ - to classical Helmholtz equation for vorticity $\nabla \times \boldsymbol{V}$. This equation has a remarkable feature, in particular, for large-scale processes time derivative from vorticity $d \nabla \times \boldsymbol{V} / d t$ is one of the main members (this feature is absent in the Euler equation of motion (1), in which inertial member $\rho d \boldsymbol{V} / d t$ is negligibly small in comparison with the other members). In the absence of certain information about the main forces (pressure gradient, gravity force), it allows for composing of prognostic equations and carrying out their numerical integration. Another important feature of the Helmholtz-Fridman equation in comparison with the Euler equation is the natural accounting of inhomogeneous effects of angular velocity of the Earth's rotation $\omega_{0}$ and geomagnetic field $\boldsymbol{H}_{\mathbf{0}}$. Finally, Eq. (5) is a basic condition of motion, in which $\nabla \times \boldsymbol{V}$ is always nonzero and contains full information about evolution of the vortices and planetary waves caused by nonconservative forces $(\nabla \times \boldsymbol{F} \neq 0)$ in the fluid. In the ionosphere, these are Coriolis forces $\boldsymbol{F}_{\boldsymbol{K}}=\rho\left[\boldsymbol{V} \cdot 2 \boldsymbol{\omega}_{\mathbf{0}}\right]$ and Ampere force $\boldsymbol{F}_{\boldsymbol{A}}=\left[\nabla \times \boldsymbol{h} \times \boldsymbol{H}_{\mathbf{0}}\right] / 4 \pi$. Equation (5), as an exact solution, contains the Rossby type classical equation for the slow planetary waves. Indeed, assuming that $V_{y}(x, t) \sim \exp \left(-i \omega t+i k_{x} x\right), \omega_{o z}(y)=\omega_{0} \sin \varphi$, $H_{o z}(z, y)=-H_{p} \sin \varphi^{\prime}, \partial / \partial y=R^{-1} \partial / \partial \varphi, \varphi-$ geographic latitude (assuming $\varphi \approx \varphi^{\prime}$ ) , $R$ - Earth's radius, $\omega$ - wave frequency, $k_{x}$ - wave number, we obtain Rossby type equation

$$
\frac{\partial^{2} V_{y}}{\partial t \partial x}+\left(\beta+\beta_{H}\right) V_{y}=0
$$

where $\beta=\left(\partial 2 \omega_{0 z} / \partial y\right)=\left(2 \omega_{0} \cos \varphi / R\right)>0$ - Rossby parameter, $\beta_{H}=-\left(e N / N_{n} M c\right)\left(\partial H_{o z}(y, z) / \partial y\right)=-\left(e N H_{p} / N_{m} M c\right)$ $(\cos \varphi / R)<0$ - "Magnetic Rossby parameter". Taking into account perturbations of the geomagnetic field $h_{z}(x, t)=\alpha \rho \partial A_{y} / \partial x$ from Eqs. (1) and (2) if $\omega_{0}=0$ we receive the set of equations for the perturbed values $V_{y}$ and $A_{y}$ :

$\frac{\partial^{2} V_{y}}{\partial t \partial x}=c_{H} \frac{\partial^{2} A_{y}}{\partial x^{2}} \quad, \quad \frac{\partial^{2} A_{y}}{\partial t \partial x}+\beta_{H} V_{y}=-\delta c_{H} \frac{\partial^{2} A_{y}}{\partial x^{2}}$

where $c_{H}=(c / 4 \pi e N)\left(\partial H_{0 z} / \partial y\right)=-\left(c H_{p} / 4 \pi e N\right)(\cos \varphi / R)$ - phase velocity of the planetary waves. Solution set of Eq. (8) (when $\delta=1$ ) gives the dispersion equation

$$
\frac{c}{c_{H}}-\frac{\beta_{H}}{c k_{x}^{2}}=1
$$


Hence, we have the high phase velocities $c=c_{+}=c_{H}$, and for the slow Rossby type waves $c=c_{R}^{\prime}=-\beta_{H} / k_{x}^{2}$. For midlatitudes in the dynamo region of the ionosphere at night $N \sim 10^{4} \mathrm{sm}^{-3}, \rho=10^{-11} \mathrm{gr} \mathrm{sm}^{-3}$, for the "fast" planetary wave we obtain $c=c_{H}=4 \mathrm{kms}^{-1}$; and for the "slow" wave $c_{R}^{\prime}=32 \mathrm{~m} \mathrm{~s}^{-1}$; in the daytime $N \sim 10^{5} \mathrm{sm}^{-3}$, $\rho=10^{-11} \mathrm{gr} \mathrm{sm}^{-3}$ and $c=c_{H}=40 \mathrm{~km} \mathrm{~s}^{-1}$. The period of the "fast" wave varies from 8 to $40 \mathrm{~min}$ at night and from 1 to $7 \mathrm{~h}$ by day when the wavelength changes from $2 \times 10^{3} \mathrm{~km}$ up to $10^{4} \mathrm{~km}$, while the time for the "slow" wave will change from 2 days to 14 days. The physical existence of these new waves in the ionosphere follows from the fact that in the dynamo region the generalized Ohm's law has the following form $\left[\boldsymbol{j} \cdot \boldsymbol{H}_{\mathbf{0}}\right] / c=e N\left(\boldsymbol{E}+\left[\boldsymbol{V} \cdot \boldsymbol{H}_{\mathbf{0}}\right] / c\right)$. Hence, we obtain for the Amphere forces $\boldsymbol{F}_{A}=\left[\boldsymbol{j} \cdot \boldsymbol{H}_{\mathbf{0}}\right] / \rho c=\left[\boldsymbol{u} \cdot 2 \boldsymbol{\Omega}_{\boldsymbol{i}}\right]$, where $\boldsymbol{u}=\boldsymbol{V}-\boldsymbol{V}_{\mathbf{0}}, \boldsymbol{V}_{\mathbf{0}}=c\left[\boldsymbol{E} \cdot \boldsymbol{H}_{\mathbf{0}}\right] / H_{0}^{2}, 2 \boldsymbol{\Omega}_{\boldsymbol{i}}=e N \boldsymbol{H}_{\mathbf{0}} / N_{n} M c$. Outwardly, the expression $\boldsymbol{F}_{A}$ coincides exactly with the Coriolic force $\boldsymbol{F}_{\boldsymbol{C}}=\left[\boldsymbol{V} \cdot 2 \boldsymbol{\omega}_{\mathbf{0}}\right]$. This similarity suggests that in the dynamo region excitation of the above waves is caused by inhomogeneity of the geomagnetic field $\left(\partial H_{0 z} / \partial y \neq 0\right)$ like the ordinary Rossby waves excite due to inhomogeneity of the angular velocity of the Earth's rotation $\left(\partial 2 \omega_{0 z} / \partial y \neq 0\right)$. At the same time, part of the electromagnetic force $\boldsymbol{F}_{A}$ caused by an electrical drift $\boldsymbol{V}_{\boldsymbol{D}}$ will generate "fast" planetary wave (with a vertical electrical field $E$ ), and part of the electromagnetic force $\boldsymbol{F}_{A}$ caused by the velocity of the flow of medium (in the dynamo region $\boldsymbol{V}_{\boldsymbol{i}} \approx \boldsymbol{V}$ ) by the dynamo field $\boldsymbol{E}_{\boldsymbol{d}}=\left[\boldsymbol{V} \cdot \boldsymbol{H}_{\mathbf{0}}\right] / c$ will generate slow planetary Rossby wave (Khantadze, 1986). For the F-region $(\delta=0)$ from Eq. (8) we obtain the equation for the standing planetary waves $\left(\partial^{2} / \partial t^{2}+\beta_{H} c_{H}\right) V_{y}=0$. Hence, we have $\omega_{n}= \pm \sqrt{\beta_{H} c_{H}}= \pm\left(H_{p} / \sqrt{4 \pi \rho}\right)(\cos \varphi / R)$ or $c_{n}=\omega_{n} / k_{x}= \pm\left(H_{p} / \sqrt{4 \pi \rho}\right)\left(\cos \varphi / R k_{x}\right)$. Frequencies $\omega_{H}$, $\omega_{p}^{\prime}$ and $\omega_{n}$ are new branches of the ionospheric resonator.

In three-dimensional approximation for the internal planetary waves, the following formulae were obtained (Khantadze et all., 2008a, b) for E-region of an ionosphere $(\delta=1)$

$\omega=\omega_{H}=\omega_{H E} \frac{\sqrt{1+3 \sin ^{2} \theta}}{R} \frac{k_{x}}{k_{x}^{2}+k_{y}^{2}+k_{z}^{2}} \quad$,

$\omega=\omega_{p}^{\prime}=\omega_{i E} \frac{\sqrt{1+3 \sin ^{2} \theta}}{R} \frac{k_{x}}{k_{x}^{2}+k_{y}^{2}+k_{z}^{2}}$.

and for F-region $(\delta=0)$

$\omega=\omega_{n}= \pm \omega_{a E} \frac{\sqrt{1+3 \sin ^{2} \theta}}{R} \frac{k_{x}}{k_{x}^{2}+k_{y}^{2}+k_{z}^{2}} \quad$,

where: $\omega_{H E}=\frac{c k^{2} H_{E}}{4 \pi e N}, \omega_{i E}=\frac{N}{N_{n}} \frac{e}{M c} H_{E}, \omega_{a E}=\frac{k H_{E}}{\sqrt{4 \pi \rho}}$ are VLF helocines for the ion-cyclotron and Alfven-type waves on the equator. In this approximation the expression for Rossby waves were obtained (Gill, 1986):

$\omega=\omega_{p}=-\frac{\beta k_{x}}{k_{x}^{2}+k_{y}^{2}+k_{z}^{2}}$.
Taking into account the compressibility and temperature stratification, it was found that (Pokhotelov et al., 2001; Onishchenko et al., 2008)

$\omega=\omega_{p}=-\frac{\beta_{0} k_{x}}{k_{x}^{2}+k_{y}^{2}+k_{z}^{2}+\left(\frac{D}{H}\right)^{2}\left(\gamma-\frac{1}{\gamma}\right)+\frac{D^{2}}{4 H^{2}}}$

which at $\frac{D}{H} \rightarrow 0, \beta_{0} \rightarrow \beta$ passes to the formula (13). From Eqs. (10-12) it follows that contrary to the Rossby-type waves (Eq. 13), these electromagnetic planetary waves propagate to the east. Formulae (10-12) show that these waves have a general planetary character and they could be registered at all longitudes from the pole to the equator. We also notice that formulae (10-12) do not take into account curvature of magnetic force lines and, in this case, as it will be shown below, the fundamental feature of magnetohydrodynamical planetary waves - anisotropic propagation of these waves along latitude - losses.

The geomagnetic field $\boldsymbol{H}_{\mathbf{0}}$ in the ionosphere is well approximated by the dipole field of a magnetized sphere. In a spherical coordinate system $(r, \theta, \lambda)$ if a dipole moment of the Earth coincides with the axis of the Earth's rotation, geomagnetic field components (Elemen, 1973):

$H_{o r}=-\frac{2 Q}{r^{3}} \cos \theta \quad, \quad H_{o \theta}=-\frac{Q}{r^{3}} \sin \theta \quad, \quad H_{0 \lambda}=0$

satisfy Maxwell's equations: $\nabla \times \boldsymbol{H}_{\mathbf{0}}=0$ and $\nabla \cdot \boldsymbol{H}_{\mathbf{0}}=0$. Components $\left(\nabla \times \boldsymbol{H}_{\mathbf{0}}\right)_{r}$ and $\left(\nabla \times \boldsymbol{H}_{\mathbf{0}}\right)_{\theta}$ identically tend to zero and these equalities give the linkage between gradients and curvature components of the geomagnetic field:

$$
\begin{aligned}
& \frac{1}{r} \frac{\partial H_{0 r}}{\partial \theta}=\frac{\partial H_{0 \theta}}{\partial r}+\varepsilon \frac{H_{0 \theta}}{r}, \\
& \frac{1}{r} \frac{\partial H_{0 \theta}}{\partial \theta}=-\frac{\partial H_{0 r}}{\partial r}-\varepsilon\left(\frac{2}{r} H_{0 r}+\frac{c t g \theta}{r} H_{0 \theta}\right) .
\end{aligned}
$$

Here parameter $\varepsilon$, as well as the parameter $\delta$, in the set of Eqs. (8) is introduced for the convenience: neglecting terms corresponding to the curvature of the geomagnetic field lines we should equal $\varepsilon=0$, if the curvature is taking into account $\varepsilon=1$. It should be noticed that a simplification of Eq. (16) using a "traditional" method $(\varepsilon=0)$, is mathematically incorrect, as in this case the geomagnetic field differs from a dipole one within accurate 20\%. Observations (Elemen, 1973) show that such a deviation of the geomagnetic field from a dipole one is revealed at a distance of ten thousand kilometers and more. Therefore, the expressions (10-12) are just approximate formulae for the phase velocities of the considered waves. Taking into account $\varepsilon=1$, from the set of Eqs. (8) (at $\delta=0$ ) we easily obtain the correct formula for the phase velocity of magneto-gradient waves simultaneously taking into account both the curvature effects and the latitudinal gradients of the geomagnetic field for F-region of an ionosphere

$c_{H}=\frac{1}{2} \frac{H_{e}}{\sqrt{4 \pi \rho}} \frac{-\sin \theta \pm \sqrt{24+\sin ^{2} \theta}}{k R}$. 
A similar expression has been obtained for formulae (10) and (11) as well (Khantadze et al., 2006). As it follows from Eq. (17), the curvature effect of the geomagnetic field causes anisotropic electromagnetic elasticity in the ionospheric plasma. As a result, electromagnetic perturbation in the F-region propagates in both east and west directions with different phase velocities. For example, as it follows from Eq. (17), on the equator $(\theta=\pi / 2)$ for wave propagating west-east direction $\left(c_{n}>0\right)$ we obtain: $c_{n+}=2 c_{0}$, where $c_{0}=H_{e} / k R \sqrt{4 \pi \rho}$. For wave propagating east-west direction $\left(c_{n}<0\right)$, we have $c_{n-}=-3 c_{0}$. Hence, we have a fundamental conclusion: geomagnetic field stratifies ionospheric plasma in the longitudinal direction $\lambda$, just as the gravity force stratifies the atmosphere at a height. Waves propagate parallel to the west faster than to the east; i.e. curvature of a geomagnetic field allocates a preferred direction for ULF electromagnetic planetary waves. Planetary waves $c_{n}$ and $c_{p}^{\prime}$ propagate to the west faster than to the east, waves $c_{H}$, vice versa, propagate to the east faster than to the west. This important feature of the magneto-gradient planetary waves allows an accurate register in the E- and F-regions of an ionosphere by ionospheric and magnetic observatories located at the same latitude. Rossby waves do not have similar features, which in principle, could be registered in the E-region where plasma particles are completely captured by neutral particles. Rossby waves in the F-region, where neutral and plasma particles have different velocities, is impossible to reveal as there are no effective methods of a wind-speed measurement in the F-region of an ionosphere.

\section{Excitation, intensification and propagation of large- scale vortices and planetary waves in the ionosphere}

Not considering any more detail in the new branches of planetary waves (see Khantadze et al., 2008; Khantadze and Jandieri, 2009), we would like to note that beginning with the altitude of $80 \mathrm{~km}$ and higher, the upper atmosphere of the Earth is a strongly dissipative medium. The vertical coefficient of turbulent mixing in the lower ionosphere (70-125 km) according to all existing estimations is of the order $10^{6} \mathrm{~m}^{2} \mathrm{~s}^{-1}$. Often when modelling large-scale processes for this region of the upper atmosphere, effective coefficient of Rayleigh friction (the last term on the rightside of the Eq. 1.2) between the ionospheric layers is introduced where the altitudes of about $100 \mathrm{~km}$ amounts to $\lambda \approx 10^{-5} \mathrm{~s}^{-1}$. The role of the "ion" friction rapidly increases at the altitudes above $120 \mathrm{~km}$ and its analytical expression coincides with the Rayleigh friction formula. Therefore, often during a study of large-scale $\left(10^{3}-10^{4} \mathrm{~km}\right)$ vortex structures and low-frequency $\left(10^{-4}-10^{-6} \mathrm{~s}^{-1}\right)$ planetary waves in the ionosphere, we will apply the well-known Rayleigh formula $\boldsymbol{F}=-\lambda_{R a} \boldsymbol{V}$ assuming the altitudes 80 $130 \mathrm{~km} \mathrm{~s} \lambda_{R a} \approx 10^{-5} \mathrm{~s}^{-1}$, and the altitudes above $130 \mathrm{~km}$ $\lambda_{R a}=N v_{i m} / N_{m}$. Dissipative force $\mathrm{F}$ has an accumulative na- ture and its action becomes perceptible only after a certain time interval $\left(t \sim 1 / \lambda_{R a}\right)$. The real mechanism of dissipation in the atmosphere against the background of baroclinic, nonlinear and dispersive effects generates in the ionosphere moving spatial structures representing the equilibrium stationary solutions of the equations of magnetic hydrodynamics. For example, the simplified Helmholtz-Fridman equation:

$$
\frac{\partial \nabla \times \boldsymbol{V}}{\partial t}=\boldsymbol{A}
$$

describes the generation of nonzero vorticity $\nabla \times \boldsymbol{V}$ in the atmosphere under the action of baroclinic vector $\boldsymbol{A}$ taking into account the temperature contrasts in the form of advection of warm and cold. According to the observations (Petersen, 1956), vector $\boldsymbol{A}$ is a slowly varying function of time and vortex Eq. (18), with the initial conditions of Cauchy $\nabla \times\left.\boldsymbol{V}\right|_{t=0}=0$ (at the initial moment in the atmosphere there were no vortices) has an unreal solution as under the action of baroclinic vector $\boldsymbol{A}$ the generating vortex will be growing unlimitedly in time $\nabla \times \boldsymbol{V}=\boldsymbol{A} \cdot t$. If we take into account Rayleigh friction, the equation for the vorticity will take the following form:

$$
\frac{\partial \nabla \times \boldsymbol{V}}{\partial t}=\boldsymbol{A}-\lambda_{R a} \nabla \times \boldsymbol{V},
$$

which with the same Cauchy conditions has the bounded solution:

$\nabla \times \boldsymbol{V}=\frac{\boldsymbol{A}}{\lambda_{R a}}\left(1-e^{-\lambda_{R a} t}\right)$.

Indeed, from Eq. (20) it follows that vorticity will increase linearly with time only at small time intervals $\left(t \ll 1 / \lambda_{R a}\right)$ under the action of baroclinicity. After a certain time, when the dissipation effect reaches a specific value, the vortex starts to decrease and for the large intervals of time $\left(t \gg 1 / \lambda_{R a}\right)$ tends to constant (equilibrium) value $\boldsymbol{A} / \lambda_{R a}$. The value $T=1 / \lambda_{R a}$ can be called a relaxation time of nonstationary vortex. For the lower atmosphere $T$ is of the order of twentyfour hours and consequently here large-scale vorticies must be long-lived (Khantadze, 1973). Stationary solution describes the equilibrium between baroclinicity and the dissipation effects $\left(\boldsymbol{A}=\lambda_{R a}(\nabla \times \boldsymbol{V})_{s t}\right)$. As a result, the dissipative structure of movement is formed in the ionosphere in the form of stationary cyclones and anticyclones. In the troposphere, such immovable cyclones (anticyclones) are called "centres of action of atmosphere" (Petersen, 1956). Against the background of such stationary structures in the ionosphere, there usually occur large-scale $\left(10^{3}-10^{4} \mathrm{~km}\right)$ and small-scale $\left(10^{-3}-10^{3} \mathrm{~km}\right)$ weather-forming nonstationary processes.

Stationary vorticity structures in the ionosphere are the consequence of invariants of Helmholtz-Fridman equations and Maxwell induction equation, taking into account Hall's effect. These equations naturally include internal, selfconsistent and opposite processes. In case of the preservation of invariant, the increase of one process is compensated 
by the decrease of another and vice-versa. Let's illustrate this from condition of invariant conservation (Eq. 5). When $\omega_{0}=0$, Eq. (5) for a plane motion is reduced to the following formula:

$\frac{d}{d t}\left((\nabla \times \boldsymbol{V})_{z}+\frac{N}{N_{n}} \frac{e}{M c} H_{0 z}\right)=0$.

Specific solution of this equation has the following form

$(\nabla \times V)_{z}+\frac{N}{N_{n}} \frac{e}{M c} H_{0 z}=A=$ const.

The geomagnetic field $H_{0 z}$ in the meridional direction has a maximum on a pole and decreases to zero on the equator. Now let's consider the case when the initial time a movement is counter-clockwise, i.e. $A>0$. Let $x$ be directed along latitude from the west to the east, and $y$ - along meridian from the south to the north. In the plane $x y$, to the left of the instant centre of rotation, and in the case of motion of particles from the north to the south, $H_{0 z}$ will decrease towards negative $y$. Consequently $\operatorname{rot}_{z} \boldsymbol{V}$ must increase by equal value so that $A$ remains constant. To the right of the instant centre of rotation, when the particles move towards positive $y, H_{0 z}$ will increase and $(\nabla \times \boldsymbol{V})_{z}$ - decrease. Thus, the presence of inhomogeneous magnetic field $H_{0 z}$ along the meridian leads to the intensification of the vortex to the left from the instant centre of rotation, i.e. particles on the left-hand side will be rotating faster than to the right of the rotation centre. In case of clockwise rotation, constant $A$ will be negative and taking this into consideration intensification of the vortex will occur again on a left-hand side from the instant centre. The intensification mechanism of such a vortex is similar to the amplification of the currents at the western ocean coasts caused by the Coriolis force (Pedlosky, 1984).

The problem of possible generation of vortical motions in the ionosphere is not less important, when at the initial time the motion is vortex-free and rectilinear along the meridian (from the north to the south). At the initial moment from the condition of retaining the Eq. (21) we will have (e $\left.N H_{0 z} / N_{n} M c\right)=A=$ const. When particles move towards negative $y, H_{0 z}$ will decrease. Consequently, positive vortex $\operatorname{rot}_{z} \boldsymbol{V}$ must occur, which will compensate the decrease of $H_{0 z}$, so that $A$ remains constant. Rotational counterclockwise motion occurs further, the results of which will be intensification of the positive vortex to the left from the instant centre of rotation and weakening to the right. Thus, we propose the simple hydrodynamic mechanism of generation of large-scale vortices in the ionosphere caused by inhomogeneity along the meridian due to the geomagnetic field of the Earth.

When studying nonstationary processes in the ionosphere, it is necessary to take into account that atmosphere density at ionospheric altitudes is nearly a million times less than in the troposphere, and the wind velocity exceeds the wind velocity in the troposphere by an order of magnitude. Therefore, ionosphere (beginning from $120 \mathrm{~km}$ and higher) becomes nonstationary and a nonlinear medium. As a rule, considering nonlinearity, steepness of the wave front increases leading to its breaking or formation of shock wave. However, as it is well known, shock waves do not arise spontaneously in the ionosphere. This indicates that in the real ionosphere for the planetary-scale motions when dissipative forces can be neglected, dispersive effects of the medium must be essential. As a result, before breaking the wave must disintegrate either into separate nonlinear waves or into the vortex formations. If nonlinear increase of the steepness of wave front will be exactly compensated by dispersion spreading, then stationary waves will appear in the ionosphere. By applying simplification of the theory of long waves and neglecting electromagnetic and viscous forces for barotropic, nondivergent atmosphere, after introduction of flow function $\psi$ by formulae $V_{x}=-\partial \psi / \partial y, V_{y}=\partial \psi / \partial x$, from Eq. (5) we get the nonlinear equation for vorticity

$$
\frac{\partial(\Delta \psi+f)}{\partial t}-\frac{\partial \psi}{\partial y} \frac{\partial}{\partial x}(\Delta \psi+f)+\frac{\partial \psi}{\partial x} \frac{\partial}{\partial y}(\Delta \psi+f)=0,
$$

where $f=2 \omega_{0} \sin \varphi-$ Coriolis parameter, $\Omega_{z}=\left(\partial V_{y} / \partial x\right)-\left(\partial V_{x} / \partial y\right)=\Delta \psi-$ vortex. Below linear and nonlinear planetary waves on the bases of Eq. (22) are investigated.

Partial solutions of Eq. (22) containing nonlinearity and dispersion can be nonlinear waves either of solitary (solitons) or periodical (knoidal) type. To show this, let's assume that $\psi=\psi(\theta, y) ; \theta=x-c t$. Then Eq. (14) will take the following form:

$J(\psi+c y ; \Delta \psi+f)=0$,

where $J(a, b)=(\partial a / \partial \theta)(\partial b / \partial y)-(\partial a / \partial y)(\partial b / \partial \theta)-$ Jacobian.

General solution of Eq. (15) has the following form

$\Delta \psi+f=G(\psi+c y)$,

where $G$ - arbitrary differentiable function of its own argument. In Kobaladze et al. (1981) we have shown that in the presence of zonal flow with small inhomogeneity along meridian $u=u_{0}\left[1+\alpha \sin \left(æ y_{0}\right)\right]$ and weak nonlinearity for the value $\delta=y_{0}-y$ from Eq. (24), the following nonlinear equation can be obtained

$$
\Delta \delta+\frac{1}{\bar{u}-c} \frac{d \bar{u}}{d y_{0}}\left[\left(\frac{\partial \delta}{\partial y}\right)^{2}+\left(\frac{\partial \delta}{\partial x}\right)^{2}+2 \frac{\partial \delta}{\partial y}\right]+\frac{\beta}{\bar{u}-c} \delta=0
$$

with homogeneous zonal flow ( $\bar{u}=$ const) Eq. (25) becomes linear and coincides with the well-known wave equation of Rossby

$$
\frac{\partial^{2} \delta_{0}}{\partial \eta^{2}}+\frac{\beta h^{2}}{\bar{u}-c_{0}} \delta_{0}=0
$$

where $\delta_{0}=\delta / a, \eta=y / h, \xi=\theta / \lambda$ - non-dimensional parameters; $a$ - wave amplitude, $h$ - thickness of a zonal flow, $\lambda-$ 
wavelength. General solution of Eq. (25) with the boundary conditions $\delta_{0}(\xi, 0)=\delta_{0}(\xi, 1)=0$ has the following form

$\delta_{0}(\xi, \eta)=F(\xi) \sin (n \pi \eta)$

$c_{0}=u_{0}-\frac{\beta}{m^{2}} \quad, \quad(n=1,2,3)$,

where $m=n \pi / h$ - wavenumber, $F(\xi)$ - arbitrary function. Solving Eq. (26) by applying theory of perturbation, in the second approximation we will get nonlinear equation for the function $F(\xi)$

$\frac{d^{2} F}{d \xi^{2}}+a_{1} F^{2}+a_{2} F=0$

Here the coefficients $a_{1}$ and $a_{2}$ are expressed with parameters $\alpha, u_{0}, a, \lambda, \beta, h, n$ and we are not giving them here because of their bulkiness. Equation (27) investigated in detail in the above-mentioned papers is a stationary equation of Korteweg-de Vries, integrated once and describes structure of nonlinear waves. Depending on integration constants, the solution represents either solitary wave (soliton) or periodical (knoidal) wave. The solutions at infinity tending to zero are of interest in meteorology (since with $\xi \rightarrow \infty, y \rightarrow y_{0}$ ) and therefore the solution for Eq. (27) has a form of solitary-type wave

$F(\xi)=A_{1} \sec h^{2}\left(A_{2} \xi\right)$,

where $A_{1}=-3 a_{2} / 2 a_{1}, A_{2}^{2}=-a_{2} / 4$. By the use of Eq. (28) current lines are constructed in a moving coordinate system $(\eta, \xi)$ for the cases $|\mathfrak{x}| \ll 1$ and $|\mathfrak{x}|=\pi$. On examination of nonlinear wave process, depending on a thickness of zonal flow, large-scale closed vortices appear in the form of cyclones and anticyclones having diameters from 500 to $1000 \mathrm{~km}$. Conditions of appearance of closed vortices in the disturbed flow have been derived. We have weak linear shift if $|æ| \ll 1$. If $æ u_{0}<0$, i.e. in case of negative linear shift, cyclonic vortex appears in the northern latitudes. If $|\mathfrak{x}|=\pi$, we have symmetrical wind shift. When $æ u_{0}<0$ where jet flows are absent, shows lines of flow of combined vortex pairs. Outwardly resembles the solution of two-dimensional solitary wave constructed in the work (Larichev and Reznik, 1976). Also it is shown that with the real atmospheric parameters, the obtained solutions make sense only for $n(n=1,2)$ corresponding to large-scale perturbations $(\lambda \sim 2 h / n)$. Such a system of solitary waves and large-scale vortices of cyclone and anticyclone-type often are observed in the atmosphere (Petersen, 1956). Thus, the presence of zonal flow inhomogeneity in meridional direction (as well as inhomogeneous geomagnetic field $H_{0 z}$ in Eq. 21) leads to formation of largescale vortices, solitary waves and jet flows, playing an important role in weather-forming processes. Since the zonal gradient of the wind velocity along the meridian always exists in the ionosphere due to uneven heating of atmosphere from the pole to the equator, caused by large-latitudinal heating source (Khantadze, 1973), the above considered mechanism of formation of nonlinear waves should play an important role in the studying of large-scale dynamic processes in the ionosphere. Therefore, the solution of dipole-type vortices should play an important role in dynamic processes of the ionosphere, since such vortical structures, as shown above, are long-living, having electromagnetic nature (this allows for the registering of them through radio methods (Sharadze et al., 1989)) and can play an essential role in the processes of heat and energy transfer and also in the formation of strongly turbulent medium.

Estimations show that (Petviashvili and Pokhotelov, 1992) energy and enstrophy of large-scale vortices in the dissipative ionosphere considerably exceed dissipative term, and accordingly relaxation of such vortices occurs very slowly. As a result, in the ionosphere in the E-region, the electromagnetic fast large-scale vortical structures are long-living (see Eq. 20). Therefore, as it was mentioned above, they can play an important role in the processes of substance, heat and energy transfer and in formation of macro-turbulent horizontal transfer, where the above vortical structures can be considered as "turbulent particles". Indeed, the abovediscussed vortical structures, playing the role of "turbulent particles", can be considered as the elements of horizontal turbulent macroexchange in the global circulation processes in the ionosphere. At the same time, horizontal turbulent exchange coefficient can be calculated by the Obukhov formula $k_{1} \simeq 10^{-2} d^{4 / 3} \mathrm{~m}^{2} \mathrm{~s}^{-1}$. For vortices of sizes $d \sim 10^{3} \mathrm{~km}$ (average size of cyclone and anticyclone) we get $k_{1} \simeq 3 \times 10^{6} \mathrm{~m}^{2} \mathrm{~s}^{-1}$. This value exceeds vertical turbulent exchange coefficient hundreds of thousands of times and shows, that during global interaction of high and low latitudes (in the ionosphere the pole is warmer than the equator) meridional transfer (from the north to the south) must have macro-turbulent character.

\section{Conclusion}

Thus, we have shown that excitation and generation of planetary-scale vortex waves in the ionosphere is caused by the permanently acting factors in the ionosphere: latitudinal gradients of the angular velocity of the Earth's rotation and geomagnetic field. To these two fundamental factors is also added the constant factor of zonal shear flow, caused by uneven heating along the meridian of polar and equator regions of ionosphere. Hence, inhomogeneity of the Earth's rotation along the meridian, geomagnetic field and zonal prevailing wind can be considered among the real sources generating planetary waves and vortex structures of an electromagnetic nature in the ionosphere.

Acknowledgements. Topical Editor M. Pinnock thanks two anonymous referees for their help in evaluating this paper. 


\section{References}

Burmaka, V. P., Kostrov, L. S., and Chernogor, L. F.: Statistical characteristics of the HF Doppler radar signals at remote sensing of the middle atmosphere by rockets starts and solar terminator, Radiophys. Radioastronomy, 8, 143-162, 2003 (in Russian).

Charney, I. G. and Drazin, B. G.: Propagation of planetary-scale disturbances from the lower into the upper atmosphere, J. Geophys. Res., 66, 83-110, 1961.

Diskinson, R. E.: Planetary Rossby waves propagation vertically through weak westerly wind guides, J. Atmos. Sci., 25, 9841002, 1968 .

Elemen, P.: Geomagnetic field, Cosmical Geophysics, edited by: Egelend, A., Houlter, O., and Omholt, A., Oslo, Bergen, Tromso: Universitets forlaget, 1973.

Gill, A.: Atmosphere - Ocean Dynamics, Academic Press, NewYork, 1986.

Kaladze, T. D., Pokhotelov, O. A., Shah, H. A., Khan, M. I., and Stenflo, L.: Acoustic-gravity waves in the Earth's ionosphere, J. Atmos. Solar-Terr. Phys., 70, 1607-1616, 2008.

Khantadze, A. G.: About invariant in the magnetohydrodynamics taking into account Hall's effect, Izv. Atmos. Ocean Phys., 8, 1308-1309, 1972.

Khantadze, A. G.: Some problems of the dynamics of conducting atmosphere. Metsniereba, Tbilisi, 267 p., 1973.

Khantadze, A. G., Aburjania, G. D., and Lominadze, J. G.: New branches of natural ULF electromagnetic oscillations of theionospheric resonator, Doklady Earth Sci., 406(1), 82-85, 2006.

Khantadze, A. G., Jandieri, V. G., and Jandieri, G. V.: Planetary waves in a rotational ionosphere, Plasma Phys. Rep., 34(6), 480485, 2008.

Khantadze, A. G., Lominadze, J. G., and Jandieri, G. V.: Internal waves in the ionosphere caused by the Earth's geomagnetic field, Doklady Earth Sci., 420(4), 672-675, 2008.
Khantadze, A. G. and Jandieri, G. V.: General planetary character of three-dimensional planetary waves propagation in lower and upper Earth atmosphere. J. Atmos. Solar-Terr. Phys., 71, 45-48, 2009.

Kobaladze, Z., Pataraya, L., and Khantadze, A. G.: About solitary Rossby waves. Izv. Atmos. Ocean Phys., 17(26) 649-653, 1981.

Larichev, V. D. and Reznik, G. M.: About two-dimensional solitary Rossby waves, Dokl. Earth Sci., 231(5) 1077-1079, 1976.

Nekrasov, A. K., Shalimov, S. L., Shukla, P. K., and Stenflo, L.: Nonlinear disturbances in the ionosphere due to acoustic gravity waves, J. Atmos. Solar-Terr. Phys., 57(7), 737-741, 1995.

Onishchenko, O. G., Pokhotelov, O. A., and Astafieva, N. M.: Generation of large-scale eddies and zonal winds in planetary atmospheres, Phys-Usp., 51(6) 577-589, 2008.

Pedlosky, J.: Geophysical Fluids Dynamics. Springer-Verlag, New York, Heidelberg Berlin, 1982.

Petersen, S.: Weather analyses and weather forecasting, vol. 1, Motion and motion systems, McGraw-Hill, 422 p., 1956.

Petviashvili, V. and Pokhotelov, O.: Solitary waves in plasma and in the atmosphere, Gordon and Beach Science publishers, OPA, Amsterdam, 1992.

Petviashvili, V. I., Pokhotelov, O. A, Shukla, P. K., and Stenflo, L.: Pancake cyclones and anti-cyclones in the ionosphere, Astrophys Space Sci., 202(2), 363-372, 1993.

Pokhotelov, O. A., Kaladze, T. O., Shukla, P. K., and Stenflo, L.: Three-Dimensional Solitary Vortex Structures in the Upper Atmosphere, Physica Scripta, 64, 245-252, 2001.

Sharadze, Z. S., Mosashvili, N. V., Pushkova, G. N., and Yudovich, L. A.: Longperiodical wavy perturbations in the upper mososophere and lower thermosphere, Geomag. Aeron., 29, 1032-1034, 1989. 